\title{
Se (IV) triggers faster Te (IV) reduction by soil isolates of heterotrophic aerobic bacteria: formation of extracellular SeTe nanospheres
}

Mini Bajaj and Josef Winter

\begin{abstract}
Background: Selenium and Tellurium have many common chemical properties as both belong to group 16 of the periodic table. High toxicities of Se and Te oxyanions cause environmental problems in contaminated soils and waters. Three strains ( $(4, C 6$ and $C 7)$ of selenite reducing and nanoparticle forming aerobic bacteria which were isolated from agricultural soils of India containing high concentrations of Se were investigated after 3.5 months of freeze-storage for their resistance against the toxic oxyanion tellurite and its reduction to non toxic elemental form $\mathrm{Te}^{\mathrm{O}}$ as well as nanoparticles formation.

Results: Strains C4, C6 and C7 reduced tellurite at maximum reduction rates of 2.3, 1.5 and $2.1 \mathrm{mg}$ Te (IV)/L/d, respectively and produced extracellular $\mathrm{Te}^{\mathrm{O}}$ nanospheres as revealed from SEM-EDX analysis. Production of extracellular Te nanospheres has been described seldom. Further, concurrent reduction of both selenite and tellurite by bacteria was examined as these toxic oxyanions are often present together in natural environments, mine tailings or wastewater from copper refining. Interestingly, bioreduction of $100 \mathrm{mg} / \mathrm{L}$ selenite in shake flasks was not much affected by the presence of $10 \mathrm{mg} / \mathrm{L}$ tellurite but tellurite reduction rate increased 13 fold with selenite in the medium. The concurrent reduction of these oxyanions resulted in rarely described bioformation of extracellular nanoparticles composed of both $\mathrm{Se}$ and Te, reported first time for aerobically growing heterotrophic non-halophilic bacterial cultures. Duganella violacienigra, the closely related strain to C4 was also found to be resistant to oxyanions of Se and Te.

Conclusions: Selenite reducing heterotrophic non-halophilic aerobic bacteria revived from 3.5 months freeze storage could successfully reduce toxic tellurite to non toxic elemental form and produced extracellular nanospheres during detoxification. Presence of relatively less toxic selenite in the medium triggers bioreduction of more toxic tellurite leading to formation of extracellular SeTe nanospheres which are sought by solar and optical recording media industry because of their excellent photovoltaic and optical properties. The bacterial cultures investigated in this study could be exploited commercially to remediate not only selenite and tellurite-contaminated soil and water but also for green synthesis of extracellular Se, Te and Se + Te nanospheres.
\end{abstract}

Keywords: Aerobes, Bioreduction, Selenite, Tellurite, Extracellular nanospheres

\footnotetext{
* Correspondence: mini.bajaj@kit.edu

Institute of Biology for Engineers and Biotechnology of Wastewater, Am

Fasanengarten, Karlsruhe Institute of Technology, Karlsruhe 76133, Germany
} 


\section{Background}

Selenium and tellurium share many common characteristics e.g. both metalloids are found with copper- and sulfur-bearing ores and exist in four oxidation states i.e. $+\mathrm{VI},+\mathrm{IV}, 0$, and $-\mathrm{II}$ in nature. Oxyanions (IV and VI) of both elements are soluble and mobile thus bioavailable and toxic. Elemental (zero valent) Te and Se are non soluble. Both Te and Se are found in relatively low abundances in earth's crust, yet high toxicities of these elements cause environmental problems in contaminated soils and waters e.g. in agricultural lands or wastewater discharges from industrial activities or mine tailings $[1,2]$. Due to an increased inclination towards biological treatments, microorganisms capable of reducing toxic oxyanions have been investigated by many researchers [3-10]. Relative to arsenic, not many studies discussing Se geo-chemical-microbial cycling in the environment have been reported and the studies focusing on Te are even less than Se. Till now, the biological function of Te and its microbial oxidation is not known. Since concentrations as low as $1 \mu \mathrm{g} / \mathrm{L}$ of tellurite are toxic to most bacteria $[11,12]$, it is especially used in selection media for Te resistant microorganisms which are often pathogens, e.g. Shiga toxin-producing E.coli $[9,13]$. Owing to their resemblance to sulfur, Te and Se disrupt the thiol chemistry within cells leading to toxicity in bacteria not resistant to these compounds [9]. In the last decade, many anaerobic bacteria capable of both selenium and tellurium reduction to non toxic forms have been isolated from extreme environments like ocean hydrothermal vents $[10,14]$ or the highly alkaline water of Monolake, California [7,8,15] but competent aerobic non-halophilic heterotrophic bacteria reducing both Se and $\mathrm{Te}$ are not yet known. Concurrent Te and Se reduction has also not been investigated for chalcogen reducing heterotrophic aerobic bacterial cultures except for a few studies describing synergistic reduction of $\mathrm{Te}+\mathrm{Se}$ by halophilic bacteria $[6,16,17]$.

As described previously [18], four strains of aerobic bacteria from Se enriched agricultural soils in India were isolated that were able to produce extracellular Se nanospheres during selenite reduction to $\mathrm{Se}^{0}$. The strains $\mathrm{C} 1$ and C4 (GenBank NCBI accession numbers JQ745646 and JQ745647) were found to be closely related to Duganella violacienigra species and strains C6 and C7 (GenBank NCBI accession numbers JQ745648 and JQ745649) were related to Agrobacterium tumefaciens species [18]. In the present study strains $\mathrm{C} 4, \mathrm{C} 6$ and $\mathrm{C} 7$ were revived after 3.5 months of freeze-storage and were investigated for their resistance against selenite as well as against tellurite. Tellurium is the next element to Se in chalcogen group 16 of the periodic table and shares many properties with each other. Scanning- and transmission electron microscopy (SEM and TEM) with energy dispersive X-ray (EDX) analysis were carried out to observe nanoparticle formation of elemental Se and Te produced as a result of detoxification. The effect of the presence of Se (IV) on Te (IV) reduction was further examined to elucidate bioremediation when multiple detrimental ions are present as it often occurs in natural environments or copper refining. Since isolate C4 was closely related to Duganella violacienigra [18] which is one of the few known species of this genus therefore, the type strain D. violacienigra DSM 15887 was also examined for its resistance against Te (IV) and Se (IV) as it has not been investigated before.

\section{Results and discussions}

\section{Selenite reduction after revival of freezed cultures}

Pure cultures of selenite-reducing aerobic bacteria that have been stored for 3.5 months in a deep freezer were revived by thawing at room temperature $\left(25 \pm 2^{\circ} \mathrm{C}\right)$ and incubation in media with glucose but without Se (IV). Initial growth of bacteria was slow but the growth rate accelerated during 3-4 successive incubations in fresh medium. After achieving a short lag phase, 5\% inoculum from overnight cultures was inoculated in fresh medium with $1 \mathrm{~g} / \mathrm{L}$ glucose monohydrate and $40 \mathrm{mg} / \mathrm{L} \mathrm{Se}$ as $133.22 \mathrm{mg} / \mathrm{L}$ of $\mathrm{Na}_{2} \mathrm{SeO}_{3} \cdot 5 \mathrm{H}_{2} \mathrm{O}(\mathrm{MW}=263.01 \mathrm{~g})$ for incubation at $27^{\circ} \mathrm{C}$. The concentration of Se was increased during successive incubations up to $250 \mathrm{mg} / \mathrm{L}$ Se (IV) or $832.62 \mathrm{mg} / \mathrm{L}$ of $\mathrm{Na}_{2} \mathrm{SeO}_{3} \cdot 5 \mathrm{H}_{2} \mathrm{O}$ in the medium. Selenite reduction was followed through ion chromatography showing gradual disappearance of Se (IV) over the days without appearance of any Se (VI) peaks indicating absence of Se (IV) oxidation. After 15.5 days of incubation $85 \%$ and $86 \%$ of $250 \mathrm{mg} / \mathrm{L}$ Se (IV) was reduced by C4 and C7, respectively, whereas $94 \%$ of Se (IV) was reduced by strain C6. Complete removal required less than 18 days (Figure 1a). Glucose in the medium was biodegraded within 2 days of incubation. More glucose was supplied in regular intervals to avoid carbon limitation during Se (IV) reduction (Figure 1b). Investigation of cultures with SEM-EDX revealed extracellular Se nanoparticles (figures not shown). Very few studies have been performed on the viability and cultivation of strains after storage of deep-freezed cultures. For revival, lengthy periods of incubation are often required, particularly for microbes that originate from oligotrophic habitats, where a non-growing or dormancy state may be the norm [19]. In their review, Joint et al. [20] also reported that transition from a 'non-growing' to a 'growing' state in a synthetic laboratory medium is critical and adaptation to growth conditions might be a very slow process. Bacterial cultures used in the present study were isolated from soil $[1,18]$ and after freeze-storage, successive long incubations were necessary to revive the capability of the bacteria to form elemental nanoparticles by adapting them again to increasing concentration of Se (IV). 

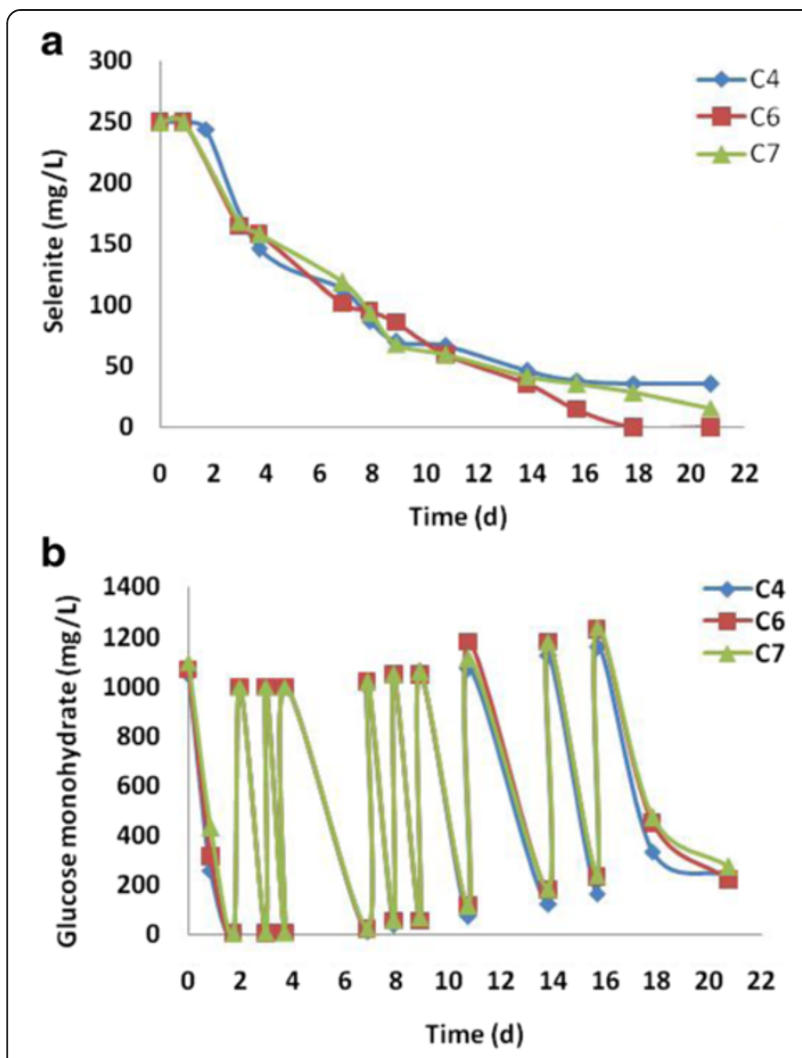

Figure 1 Selenite reduction efficiency of bacteria. Selenite reduction (a) and glucose degradation (b) by aerobic cultures of soil isolates $\mathrm{C4}, \mathrm{C6}$ and C7 after revival from freeze-storage.

\section{Incubation with Tellurite}

Before conservation of cultures by freezing for 3.5 months, cultures had been incubated with tellurite. Within 2-3 days of incubation a change in color of the medium from colorless to black was observed (not shown). This visible observation was taken as an indication of reduction of colorless tellurite to its black colored elemental form Te (0). Since these cultures were able to retain their capacity to reduce selenite and to produce extracellular $\mathrm{Se}^{0}$ nanoparticles even after 3.5 months of freeze-storage (see above section), they were incubated with $45.75 \mathrm{mg} / \mathrm{L}$ of $\mathrm{K}_{2} \mathrm{TeO}_{3}$ $(23 \mathrm{mg} / \mathrm{L} \mathrm{Te})$ to investigate their Te (IV) reduction capability after revival. In all assays the medium changed from colorless to black and darkened up towards the end of incubation. Average Te (IV) reduction observed in the assays of strains C4, C6 and C7 after 24 days of incubation was $88 \%, 74 \%$ and $82 \%$, respectively, with maximum removal rates of 2.3, 1.5 and $2.1 \mathrm{mg}$ Te (IV)/L/d (Figure 2a). In parallel, quick glucose degradation was observed in all assays requiring re-feeding of glucose at regular intervals (Figure 2b). It was assumed that if these strains could reduce Te (IV), then they might also form Te (0) nanoparticles in analogy to Se nanoparticle formation, mimicking the Se detoxification mechanism. Therefore, SEM analyses of these cultures along with respective controls were carried out. All three Strains C4, C6 and C7 could produce extracellular $\mathrm{Te}$ nanospheres, respectively which were confirmed with EDX analysis. The nanoparticles produced by e.g. strain $\mathrm{C} 4$ are shown in Figure 2c, $d$ and e. The size of Te nanoparticles formed by the three strains ranged from ca. 33 to $220 \mathrm{~nm}$ with the majority of them having a diameter around $100 \mathrm{~nm}$. As a p-type semiconductor tellurium nanomaterials have a wide range of applications in photovoltaic cells, light-emitting diodes, sensors and nano devices due to their good photoconductivity, thermoelectricity, catalysis, high piezoelectricity, nonlinear optical properties and ability to form functional materials with many chemicals $[21,22]$. Successful application of such semiconductors depends on their morphology, structure, size and surface functionality. Therefore, new routes for manufacturing various Te nanostructures e.g. nanotubes, nanowires and nanospheres are being searched for [21].

Bacterial reduction of tellurite and its deposition occur almost exclusively intracellular [9,12,23]. Bioformation of extracellular Te nanoparticles is not common and almost all reports describe extracellular deposits as nanorods or needle like nanoprecipitates [4,5,24]. The formation of extracellular Te nanospheres was only described by the Te (IV) respiring anaerobic Sulfurospirillum barnesii [7]. This strain also reduced Se (IV) and other metalloids as terminal electron acceptors $[7,8]$. Production of extracellular spherical Te nanoparticles by heterotrophic aerobic bacteria has not yet been reported. Only a few studies described extracellular Te deposition by aerobic bacteria as Te nano rods [25] or nanoparticles of up to $300 \mathrm{~nm}$ with an undefined shape [3]. Recently, Borghese et al. [26] described reduction of Te (IV) and Se (IV) oxyanions into extracellular needle like $\mathrm{Te}^{0}$ and globular $\mathrm{Se}^{0}$ nano precipitates during anaerobic growth of the facultative photosynthetic bacterium Rhodobacter capsulatus. The formation of extracellular nano precipitates was dependent on the carbon source that was supplied for bacterial growth. The rate of chalcogen reduction was constant at different concentrations of lawsone that was added as a redox mediator to facilitate the formation of external Te nanoparticles. In the present study, no external redox mediator was required but glucose as a carbon source was essential and effective for both Se (IV) and Te (IV) reduction leading to spherical nanoparticles of Se (0) and Te (0) [[18]; Figure 2d and e) which is reported first time for aerobically growing heterotrophic non-halophilic bacterial cultures. Extracellular nanoparticles could be separated from bulk media and might be used for possible applications where a high surface to volume ratio of spherical shape is advantageous. 


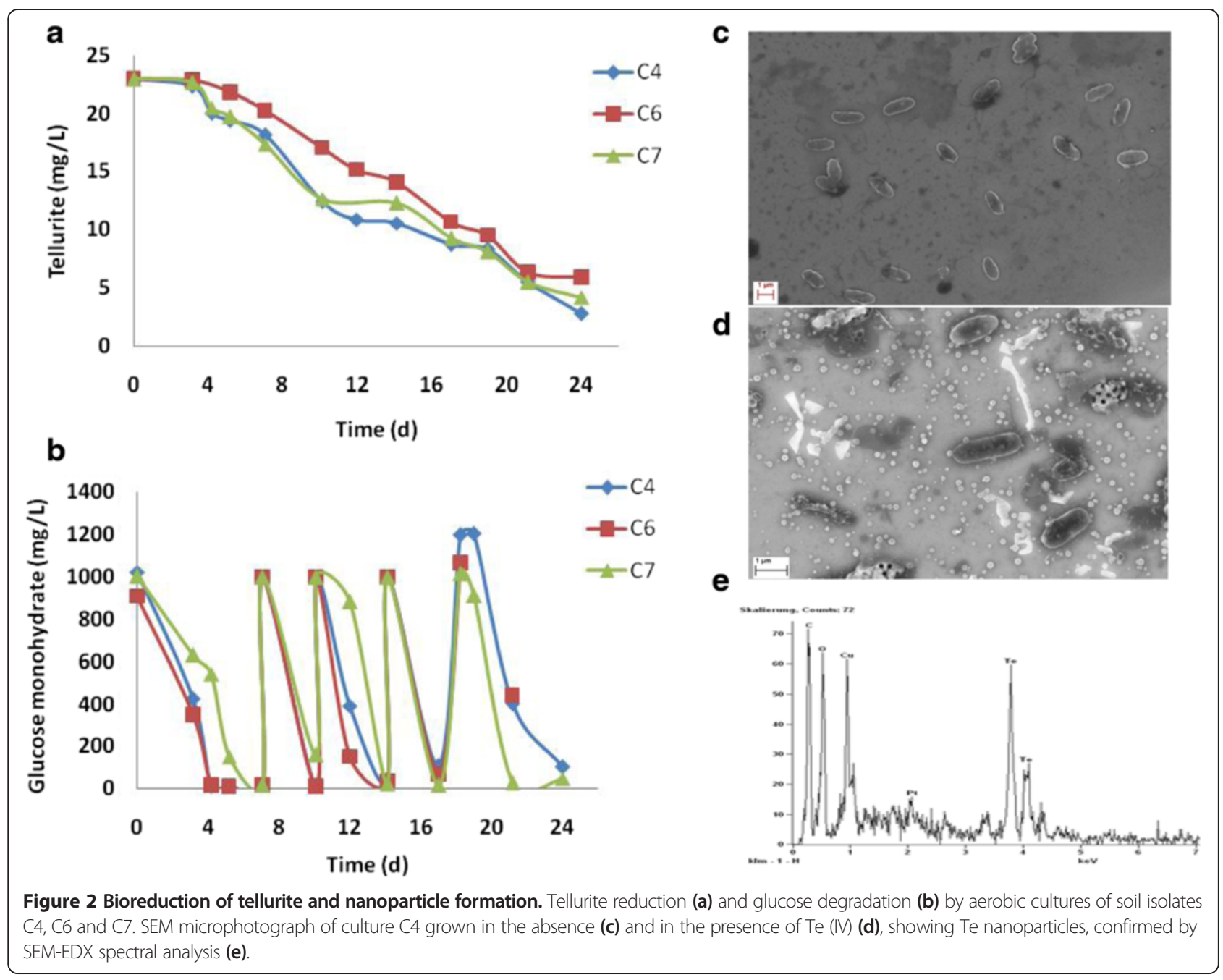

\section{Incubation of cultures in the presence of both Selenite and Tellurite}

In the previous experiments (see above section) it was confirmed that isolates C4, C6 and C7 were capable of reducing tellurite which is approximately 100 -fold more toxic to bacteria than selenite $[9,27]$. To investigate combined ion detoxification, strains $\mathrm{C} 4, \mathrm{C} 6$ and $\mathrm{C} 7$ were incubated in the presence of both Se and Te oxyanions. In the presence of $100 \mathrm{mg} / \mathrm{L}$ of Se (IV), the three strains reduced $14 \mathrm{mg} / \mathrm{L}$ of $\mathrm{Te}$ (IV) in 1.2 days (Figure 3a) and almost $100 \mathrm{mg} / \mathrm{L} \mathrm{Se}$ (IV) in about 9 days (Figure 3b) during regular re-feeding of $1 \mathrm{~g} / \mathrm{L}$ glucose after its degradation (Figure 3c). The control assay that contained only $14 \mathrm{mg} / \mathrm{L}$ of Te (IV) and no Se (IV) required a longer time for a non-complete reduction of tellurite (Figure 3d) indicating that Te (IV) reduction was stimulated by parallel reduction of a similar amount of Se (IV). Selenite reduction in the presence or absence of Te (IV) occurred at about the same rate (Figure 3b, e). Reduction of chalcogens was not observed in sterile controls which was also confirmed by lack of color change in the medium (Figure 3 inset). On the fourth day of incubation, samples were taken for SEM- and TEM-EDX analysis to investigate formation and composition of nanoparticles. When the samples were taken, $100 \%$ of $14 \mathrm{mg} / \mathrm{L}$ Te (IV) and $67.7 \%$, $73.3 \%$ and $73.0 \%$ of $100 \mathrm{mg} / \mathrm{L}$ Se (IV) in the medium was reduced by the cultures of strains $\mathrm{C} 4, \mathrm{C} 6$ and $\mathrm{C}$, respectively. With SEM, spherical nanoparticles with a diameter ranging from 50 to $150 \mathrm{~nm}$ were observed in culture C4, 70 to $140 \mathrm{~nm}$ in culture C6 and 86 to $131 \mathrm{~nm}$ in culture C7. The majority of nanoparticles formed by cultures $\mathrm{C} 4$, C6 and C7 had a diameter of $100 \mathrm{~nm}$ (Figure 4a). SEMEDX analyses indicated Te and Se to be the elemental constituents of these particles. Since SEM-EDX analyses covered an area of about $100 \mathrm{~nm}$, more precise TEM-EDX analyses with a spot size of $12 \mathrm{~nm}$ were performed. Spectra of nanoparticles of the three cultures (Figure $4 \mathrm{~b}$ ) again confirmed that each particle consisted of both Se (0) and $\mathrm{Te}(0)$. The $\mathrm{Cu}$ and Be peaks in the spectra (Figure $4 \mathrm{~b}$ ) were due to the instrumental contamination caused by the copper grid used as specimen stage and the beryllium window in TEM-EDX. No other study except the one by 


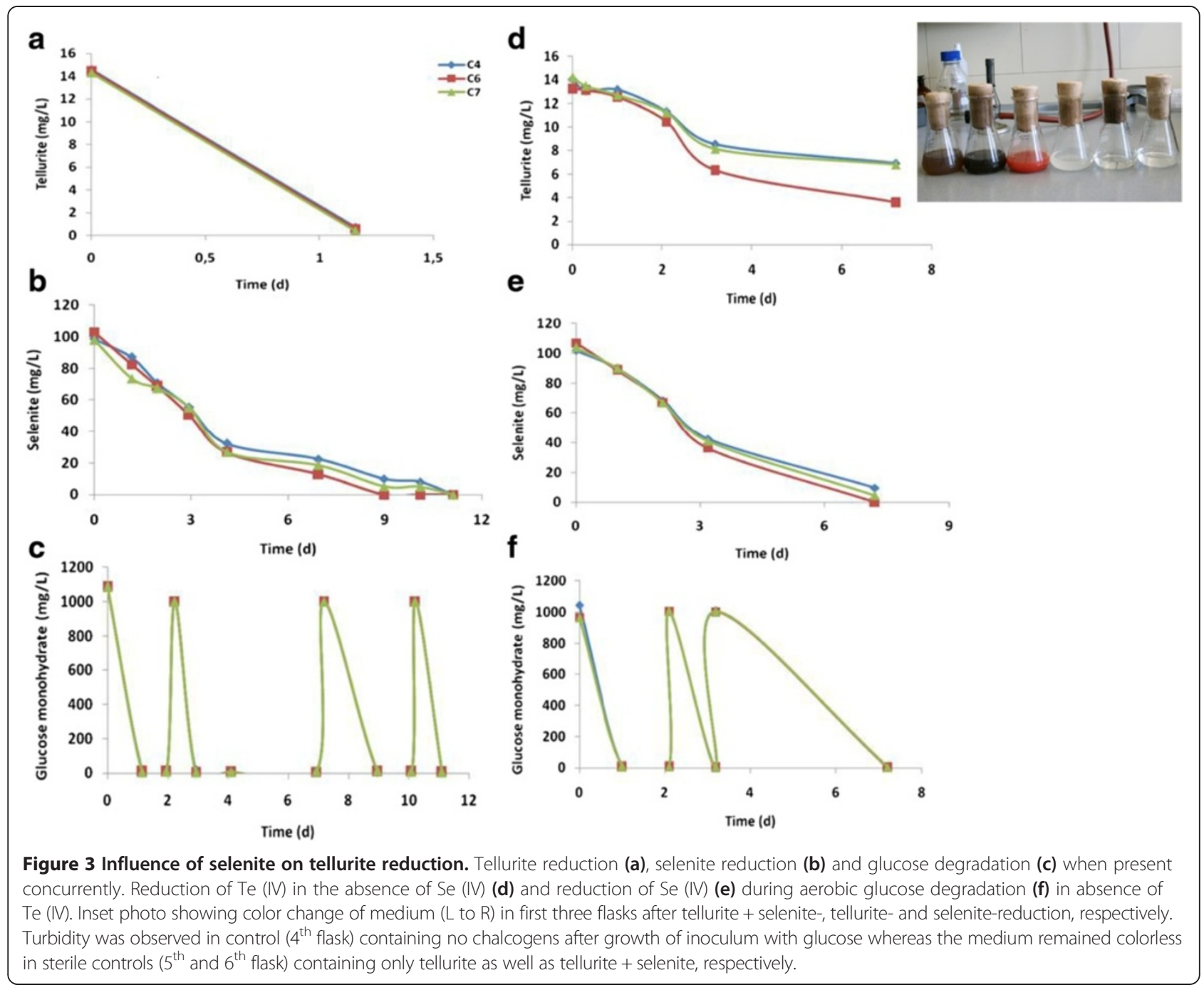

Pearce et al. [5] reported bioformation of nanospheres composed of both semi-conductive metalloids, $\mathrm{Se}+\mathrm{Te}$. In the study of Pearce et al. [5] B. beveridgei strain MLTeJB reduced up to $10 \mathrm{mM}$ Te (IV) to Te (0) as the sole reduction product under anaerobic conditions, when excess lactate was present. Subsequent addition of $5 \mathrm{mM}$ of Se (IV) to the suspension after Te (IV) reduction to Te (0) resulted in reduction of Se (0) to Se (-II). SEM-EDX analysis revealed that cells grown first in the presence of $\mathrm{Te}$ (IV) and then with Se (IV) upon depletion of the former were encrusted with nanospheres composed of both Se and Te. The mechanism of such nanoparticle formation was not clear but it was assumed that the addition of Se (IV) to the Te (0) nanoparticles containing suspension stimulated cells of $B$. beveridgei strain MLTeJB to maintain a reducing environment in which further reduction of $\mathrm{Te}(0)$ to $\mathrm{Te}(-\mathrm{II})$ was possible. In the present study, Se (IV) was added concurrently with Te (IV) to the medium and both metalloids were at the same oxidation state at start. The advantage of $\mathrm{Se}+\mathrm{Te}$ nanoparticles compared to elemental Se would be that amorphous SeTe nanostructures have greater hardness, higher crystallization temperature, higher photosensitivity and less ageing effects. As a consequence, they have several applications in optical recording media because of their excellent laser writer sensitivity, xerography, electrographic applications such as photoreceptors in photocopying and laser printing, infrared spectroscopy as well as switching and memory devices [28]. Se and Te oxyanions in the group of chalcogens can be reduced by both, anaerobic and aerobic bacteria. The majority of these reduction processes form chalcogencontaining organics e.g. volatile organometalloids and may produce elemental precipitates or nanoparticles through similar mechanisms via (1) enzymatic reduction, (2) methylation, (3) dissimilatory reduction concomitant with sulfate reduction, (4) Painter-type reactions with the thiols of proteins as well as glutathione and (5) a chemical reaction with the siderophore thiocarboxylic acid and the products of its hydrolysis $[9,29]$. Turner et al. [11] proposed a mechanism of tellurite toxicity in which tellurite oxyanions enter a cell 


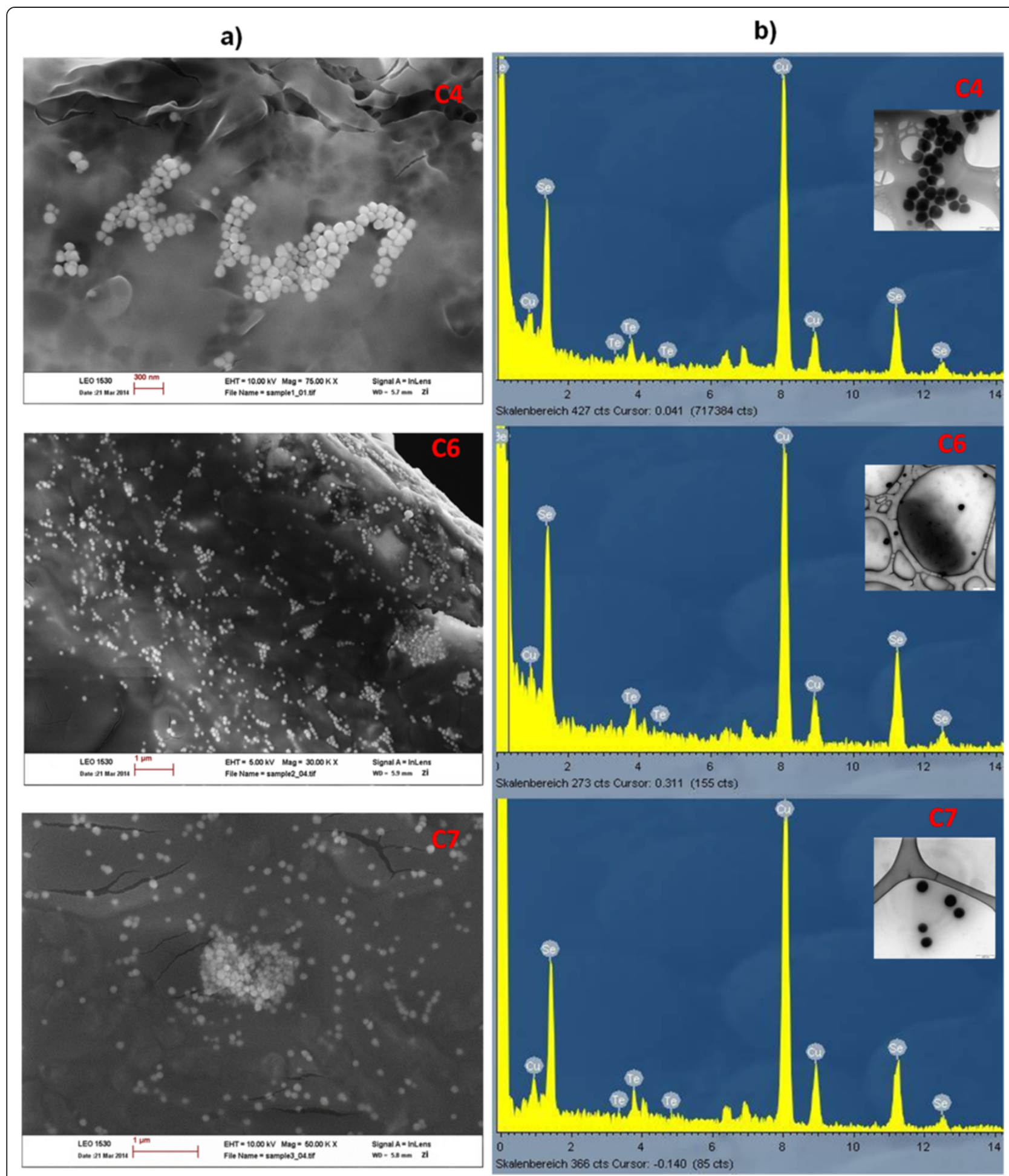

Figure 4 Extracellular production of SeTe nanospheres. SeTe nanospheres synthesized by cultures C4, C6 and C7 (a) along with respective TEM-EDX spectra (b). Inset showing TEM microphotographs of nanospheres on carbon coated copper grids.

via uptake by a phosphate transport system. Subsequently they were reduced to elemental tellurium by glutathione or other reduced thiols. Reactive oxygen species $\left(\mathrm{O}_{2}^{-}\right)$produced during reduction of tellurite may be detoxified by superoxide dismutase of aerobic bacteria. A similar mechanism was proposed by Kessi and Hanselmann [30] for selenite reduction in the glutathione synthesizing cyanobacteria and in $\alpha, \beta$, and $\gamma$ groups of proteobacteria 
(Gram-negative bacteria), thus being incompatible with strict anaerobes that lack oxidative stress enzymes. In the present study, all test strains were Gram negative proteobacteria. Strain C4 was phylogenetically closely related to $\beta$ Proteobacteria, Duganella violacienigra spec. whereas, strains C6 and C7 were closely related to $\alpha$ Proteobacteria, Agrobacterium tumefaciens spec. [18]. The presence of Se oxyanions might have triggered glutathione reductase and other enzymes required to counteract Te (IV) and reactive oxygen species. A positive effect of Se oxyanions on tellurite reduction has previously only been reported by Amoozegar et al. [6] who observed a $25 \%$ increase in the removal of $0.5 \mathrm{mM}$ tellurite from a $10 \% \mathrm{NaCl}$ containing nutrient broth in the presence of $6 \mathrm{mM}$ sodium selenite by a halophilic bacterium, Salinicoccus strain QW6, which was isolated from salty environments of Iran. In addition, in their recent study [16], improved reduction of $0.1 \mathrm{mM}$ and $0.2 \mathrm{mM} \mathrm{Te}$ (IV), respectively by halophilic Halomonas elongata DSM2581 and halotolerant Enterococcus faecalis PTCC1237 was reported in the presence of Se (IV). Increased Te (IV) reduction efficiency in presence of Se (IV) particularly by halophilic or halotolerant bacteria was assumed to be due to the presence of sodium and potassium cations in tellurite and selenite solutions, which were necessary not only for growth and survival of the bacteria but also for enzymatic activities. Alternatively, it might be due to tellurite reductase induced by selenite ions $[6,16,17]$. However, in the present study of non-halophilic/halotolerent aerobic bacteria, Se (IV)-triggered Te (IV) reduction might be due to an increased reductase activity rather than due to an effect on $\mathrm{Na}^{+} / \mathrm{K}^{+}$-ATPase influx.

\section{Tellurite reduction at various selenite concentrations and} effect of tellurite re-feeding

No Se (IV) or Te (IV) reduction was observed in assays when both oxyanions were present simultaneously at concentrations of $100 \mathrm{mg} / \mathrm{L}$ each in the medium. However, in the presence of $10 \mathrm{mg} / \mathrm{L} \mathrm{Te}$ (IV) and $100 \mathrm{mg} / \mathrm{L} \mathrm{Se}$ (IV) the maximum reduction rate of tellurite was $29.41 \mathrm{mg} \mathrm{Te}$ (IV)/Ldd (data not shown). Reduction of Te (IV) was greatly influenced by the presence of excess Se (IV). To investigate the effect of the concentration of Se (IV), $10 \mathrm{mg} / \mathrm{L}$ of Te (IV) was incubated with 5 to $40 \mathrm{mg} / \mathrm{L}$ of Se (IV) representing ratios of 2:1, 1:1, 1:2 and 1:4 Te (IV):Se (IV), respectively in the medium. Except for controls, media were inoculated with test strain C6. It was observed that Te (IV) reduction was fastest in the assays with the highest Se (IV) concentration, and Te (IV) reduction rates decreased in the order of decreasing Se (IV) concentrations (Figure 5a). It took just one day for removal of $10 \mathrm{mg} / \mathrm{L} \mathrm{Te}$ (IV) when Se (IV) was present in a ratio of 1:4 whereas in a parallel assay 8 days were required for reduction of a similar amount of Te (IV) when the Te (IV):Se (IV) ratio was 2:1. No significant change in concentrations of both Se and $\mathrm{Te}$ (measured at

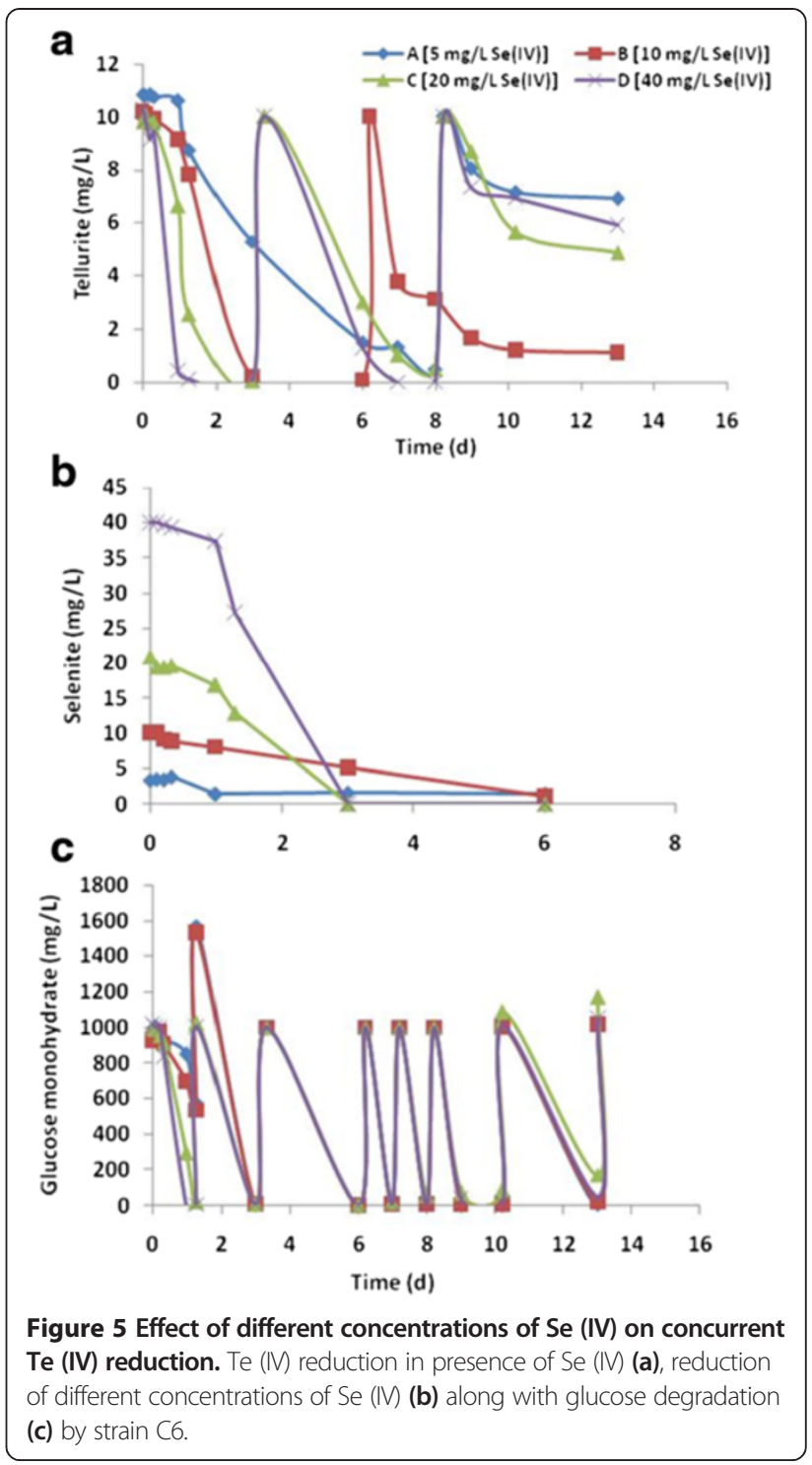

start and at the end of experiments) or the change in media color was observed in sterile controls omitting the possibility of chalcogen reduction via abiotic processes. It was obvious that Se (IV) oxyanions induced detoxification of Te (IV) resulting in faster reduction of tellurite. When the concentration of selenite was low, a quick reduction of Te (IV) for detoxification was not observed, resulting in partial inhibition of growth of the bacteria due to toxicity of Te (IV) anions in the medium. Apparently, Se (IV) reduction rates were much slower in assay with low Se (IV) concentrations than in the assays with much higher concentrations of Se (IV) (Figure 5b). After complete reduction of Te (IV) and Se (IV) in assays C and D (Figure $5 \mathrm{a}$ and b), $10 \mathrm{mg} / \mathrm{L}$ of Te (IV) was fed again in both assays. Even after complete Se (IV) reduction in these assays, the Te (IV) reduction rates at successive re-feed were much faster compared to assays incubated with Te (IV) only (Figure 3d). During this 
period glucose was re-fed twice after complete reduction of the second feeding of $10 \mathrm{mg} / \mathrm{L}$ of Te (IV) in assay D and in assay $\mathrm{C}$ (Figure $5 \mathrm{a}$ and $\mathrm{c}$ ). At the time of the third feeding of $10 \mathrm{mg} / \mathrm{L}$ of Te (IV) in assay C and D (Figure 5a), the bacteria were growing already without both oxyanions for a while and apparently have inactivated detoxification enzymes resulting in slower tellurite reduction comparing to first two feedings.

Tellurite and Selenite reduction by Duganella violacienigra In our previous study, strain C4 was identified to be $95 \%$ phylogenetically similar to Duganella violacienigra [18]. Duganella spec. are members of $\beta$-Proteobacteria within the family Oxalobacteraceae, comprising Gram-negative strictly aerobic/anaerobic bacteria. Only a few bacteria are classified within this genus [31] and their tolerance to toxic oxyanions of selenite or tellurite is not yet known. To investigate selenite and tellurite resistance, D. violacienigra DSM 15887 was grown in the absence and in the presence of $20 \mathrm{mg} / \mathrm{L}$ of Se (IV) and Te (IV), respectively. After 3 days of incubation a color change from colorless to violet was observed in control assays without Se (IV) or Te (IV) and to red or black in the medium containing Se (IV) or Te (IV), respectively. This indicated oxyanions reduction to their respective elemental forms (Figure 6). Duganella species isolated from agricultural soils $[18,31]$ and presumably other soil bacteria thus might be playing a significant role in Se and Te cycling in soil and contribute to oxyanion detoxification by reducing selenite or tellurite even under aerobic growth conditions. These microorganisms could be exploited commercially for bioreduction of toxic oxyanions of $\mathrm{Te}$

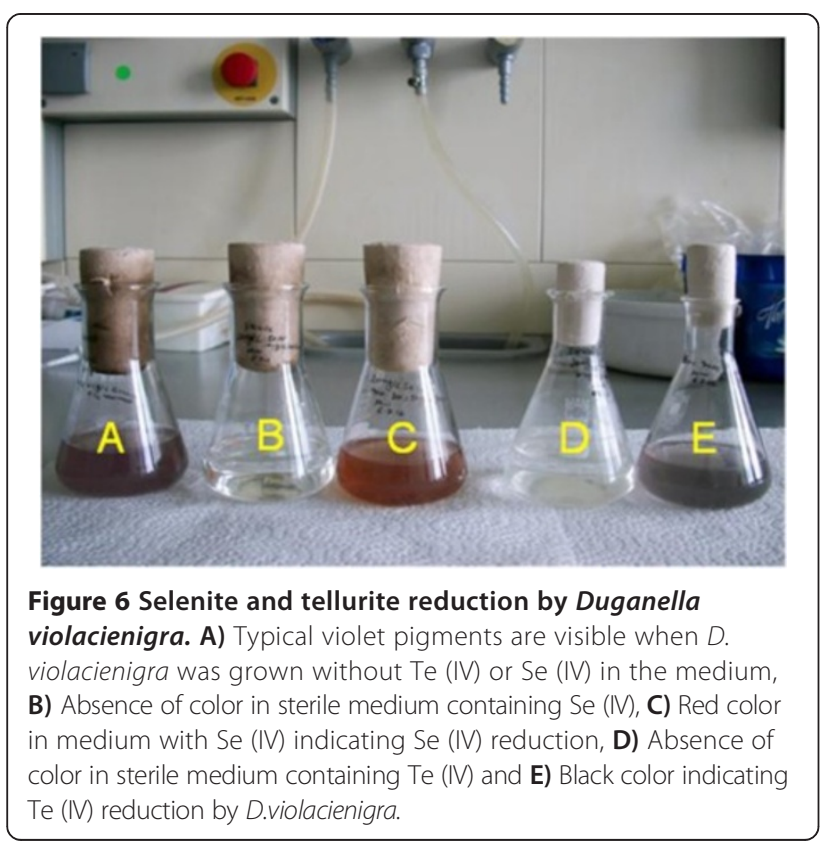

and Se to remediate selenite and/or tellurite-contaminated soil and water.

\section{Materials and methods Microbial cultures}

Isolation and identification of bacteria C4, C6 and C7 and their main physicochemical properties have been described in detail previously [18]. All bacteria were isolated from mixed microbial aerobic cultures enriched from a high selenium containing soil obtained from agricultural fields in India. After log-phase growth of the pure cultures in medium without Se (IV) (according to Ghosh et al. [32]), the cell suspension was mixed with $86 \% \mathrm{w} / \mathrm{v}$ glycerol in the ratio of $3: 1$ and stored at $-20^{\circ} \mathrm{C}$ in a deep freezer. After 3.5 months of storage the cultures were thawed and $2 \mathrm{ml}$ of respective cultures were suspended in $20 \mathrm{ml}$ medium containing $1 \mathrm{~g} / \mathrm{L}$ glucose monohydrate and no Se (IV) in $100 \mathrm{ml}$ shake flasks with cotton plugs. Flasks were incubated at $27^{\circ} \mathrm{C}$ on a rotary shaker at $110 \mathrm{rpm}$. These bacteria were adapted to high Se (IV) concentrations in successive incubations in fresh medium (40 ml) containing $1 \mathrm{~g} / \mathrm{L}$ glucose monohydrate and increasing concentrations of Se (IV) with 30-40 mg/ $\mathrm{L}$ increments during 1 to 1.5 months.

\section{Experimental design}

\section{Chalcogens resistance assays}

Growth medium [32] containing $0.03 \mathrm{~g} \mathrm{NH}_{4} \mathrm{Cl}, 0.01 \mathrm{~g}$ $\mathrm{NaCl}, 0.01 \mathrm{~g} \mathrm{MgSO}_{4}, 0.15 \mathrm{~g}$ yeast extract and $0.5 \mathrm{~g}$ peptone per $\mathrm{L}$ of deionized water, $\mathrm{pH} 7.5$ supplemented with $1 \mathrm{~g} / \mathrm{L}$ glucose monohydrate and the indicated concentrations of Se (IV) as $\mathrm{Na}_{2} \mathrm{SeO}_{3} \cdot 5 \mathrm{H}_{2} \mathrm{O}$ or Te (IV) as $\mathrm{K}_{2} \mathrm{TeO}_{3}$ (SigmaAldrich, Taufkirchen or Serva, Heidelberg, Germany) in $100 \mathrm{ml}$ Erlenmeyer flasks was inoculated with 5\% of pure cultures of the bacterial strains $\mathrm{C} 4, \mathrm{C} 6$ and $\mathrm{C} 7$, respectively. Concentrated solutions of each $\mathrm{C}_{6} \mathrm{H}_{12} \mathrm{O}_{6} \cdot \mathrm{H}_{2} \mathrm{O}$, $\mathrm{Na}_{2} \mathrm{SeO}_{3} \cdot 5 \mathrm{H}_{2} \mathrm{O}$ and $\mathrm{K}_{2} \mathrm{TeO}_{3}$, respectively were sterilized separately and added to growth medium under sterile conditions as required. Controls containing inoculum and no chalcogens as well as sterile controls containing Se (IV), Te (IV) or both Se (IV) and Te (IV), respectively were carried out for all assays. Flasks were closed with cotton plugs and incubated at $27 \pm 2^{\circ} \mathrm{C}$ on a rotary shaker at $110 \mathrm{rpm}$. Working volumes of the assays were 40 to $60 \mathrm{ml}$ and the bacterial concentration was about 1.8 to $2.2 \times 10^{7}$ cells $/ \mathrm{ml}$ in the flasks at the start of incubation.

\section{Growth of Duganella violacienigra with Te and Se}

A culture of D. violacienigra (DSM 15887) also known as Pseudoduganella violaceinigra [33] was purchased from Deutsche Sammlung für Mikroorganismen und Zellkulturen (DSMZ), Braunschweig, Germany. A loop full of preserved bacteria was inoculated in $100 \mathrm{ml}$ 
Erlenmeyer flasks containing $50 \mathrm{ml}$ medium [32] with $0.5 \mathrm{~g} / \mathrm{L}$ glucose monohydrate and incubated at $30^{\circ} \mathrm{C}$ on a shaker at $110 \mathrm{rpm}$. After appearance of the violet pigmentation, $10 \%$ of inoculum from this culture was transferred into fresh medium containing $20 \mathrm{mg} / \mathrm{L}$ of Se (IV) or Te (IV), respectively and $1 \mathrm{~g} / \mathrm{L}$ glucose monohydrate. Conditions for incubation were same as described above. Control assays with only bacteria but without $\mathrm{Te}$ or $\mathrm{Se}$ and two sterile assays containing Se (IV) and Te (IV), respectively, in the medium were run in parallel.

\section{Analyses}

Selenate and selenite were determined by ion-exchangechromatography (ICS 90, Dionex) as described earlier [18]. Tellurite in the samples was measured according to Turner et al. [34] by binding of Diethyldithiocarbamate (DDTC) with Te (IV) in the presence of Tris- $\mathrm{HCl}$ buffer and analyzing absorbance at $340 \mathrm{~nm}$ with a spectrophotometer. Glucose was determined with dinitrosalicylic acid-reagent according to Miller [35] by measuring the absorbance with a spectrophotometer at $550 \mathrm{~nm}$. Nanoparticle formation was observed and characterized by electron microscopy equipped with energy dispersive spectroscopy as described previously [18]. All chemicals used for analyses and medium were purchased from Carl-Roth, Karlsruhe, Germany except where stated otherwise.

\section{Conclusions}

After 3.5 months of freeze-storage, bacterial cultures C4, C6 and C7 isolated from soil, were revived and could retain their selenite reduction activities for soil detoxification. Beside selenite they also reduced tellurite and formed extracellular Te (0) nanospheres, which is a rare phenomenon during aerobic growth. Not much is known about detoxification of selenite and tellurite in aerobic conditions, while more information exists on the anaerobic respiration of these oxyanions. It was also possible to reduce both oxyanions concurrently and the presence of selenite induced much faster tellurite reduction rates. Owing to their similar structure and chemical properties, selenite might have triggered the uptake and reduction of more toxic tellurite during detoxification mechanism by bacteria. The optimum ratio of selenite : tellurite for faster tellurite reduction was 4:1. The three soil isolates could also produce rarely described extracellular nanospheres composed of both, $\mathrm{Se}+\mathrm{Te}$. Duganella violacienigra that is phylogenetically very close to strain $\mathrm{C} 4$, could also reduce both oxyanions. The capability of reducing selenite and tellurite particularly in aerobic conditions makes these strain worthy of possible exploitation in bioremediation as well as for environment friendly production of useful nanoparticles.
Competing interests

The authors declare that they have no competing interests.

\section{Authors' contributions}

MB designed and carried out the research work and drafted the manuscript. JW was involved in the discussions of the research results and contributed to finalize the manuscript. Both authors have read and approved the final manuscript.

\section{Acknowledgements}

Mr. Volker Zibat, Mr. Mohammed Fotouhi Ardakni and Mr. Peter Pfundstein from Laboratory of Electron Microscopy (LEM), KIT are kindly acknowledged for their assistance in electron microscopy.

Received: 1 August 2014 Accepted: 17 November 2014

Published online: 26 November 2014

\section{References}

1. Bajaj M, Eiche E, Neumann T, Winter J, Gallert C: Hazardous concentrations of selenium in soil and groundwater in North-West India. J Hazard Mater 2011, 189:640-646.

2. Harada T, Takahashi Y: Origin of the difference in the distribution behavior of tellurium and selenium in a soil-water system. Geochim Cosmochim Ac 2009, 72:1281-1294.

3. Kagami T, Fudemoto A, Fujimoto N, Notaguchi E, Kanzaki M, Kuroda M, Soda S, Yamashita M, Ike M: Isolation and characterization of bacteria capable of reducing tellurium oxyanions to insoluble elemental tellurium for tellurium recovery from wastewater. Waste Biomass Valor 2012, 3:409-418.

4. Turner RJ, Borghese R, Zannoni D: Microbial processing of tellurium as a tool in biotechnology. Biotechnol Adv 2012, 30:954-963.

5. Pearce Cl, Baesman SM, Blum JS, Fellowes JW, Oremland RS: Nanoparticles formed from microbial oxyanion reduction of toxic group 15 and group 16 metalloids. In Microbial Metal and Metalloid Metabolism: Advances and Applications. Edited by Stolz JF, Oremland RS. Washington DC: ASM press; 2011:297-319.

6. Amoozegar MA, Ashengroph M, Malekzadeh F, Razavi MR, Naddaf S, Kabiri $\mathrm{M}$ : Isolation and initial characterization of the tellurite reducing moderately halophilic bacterium, Salinicoccus sp. strain QW6. Microbiol Res 2008, 163:456-465.

7. Baesman SM, Stolz JF, Kulp TR, Oremland RS: Enrichment and isolation of Bacillus beveridgei sp nov, a facultative anaerobic haloalkaliphile from Mono Lake, California, that respires oxyanions of tellurium, selenium, and arsenic. Extremophiles 2009, 13:695-705.

8. Baesman SM, Bullen TD, Dewald J, Zhang DH, Curran S, Islam FS, Beveridge $\mathrm{TJ}$, Oremland RS: Formation of tellurium nanocrystals during anaerobic growth of bacteria that use Te oxyanions as respiratory electron acceptor. Appl Environ Microbiol 2007, 73:2135-2143.

9. Zannoni D, Borsetti F, Harrison JJ, Turner RJ: The bacterial response to the chalcogen metalloids Se and Te. Adv Microb Physiol 2008, 53:1-71.

10. Csotonyi JT, Stackebrandt E, Yurkov V: Anaerobic respiration on tellurate and other metalloids in bacteria from hydrothermal vent fields in the eastern Pacific Ocean. Appl Environ Microbiol 2006, 72:4950-4956.

11. Turner RJ, Weiner JH, Taylor DE: Tellurite-mediated thiol oxidation in Escherichia coli. Microbiology 1999, 145:549-557.

12. Yurkov $V$, Jappe J, Vermeglio A: Tellurite resistance and reduction by obligatory aerobic photosynthetic bacteria. Appl Environ Microbiol 1996, 62:4195-4198.

13. Hiramatsu R, Matsumoto M, Miwa Y, Suzuki Y, Saito M, Miyazaki Y: Characterization of Shiga toxin-producing Escherichia coli $\mathrm{O} 26$ strains and establishment of selective isolation media for these strains. J Clin Microbiol 2002, 40:922-925.

14. Rathgeber C, Yurkova N, Stackebrandt E, Beatty JT, Yurkov V: Isolation of tellurite- and selenite-resistant bacteria from hydrothermal vents of the Juan de Fuca Ridge in the Pacific Ocean. Appl Environ Microbiol 2002, 68:4613-4622.

15. Oremland RS, Herbel MJ, Blum JS, Langley S, Beveridge TJ, Ajayan PM, Sutto $T$, Ellis AV, Curran S: Structural and spectral features of selenium nanospheres produced by Se-respiring bacteria. Appl Environ Microbio/ 2004, 70:52-60.

16. Motesharrei ZS, Amoozegar MA: Bioremediation of tellurite in presence of toxic oxyanions by halophilic and halotolerant bacteria. Adv Agr Sci Eng Res 2014, 4:1437-1444. 
17. Kabiri M, Amoozegar MA, Tabebordbar MS, Gilany K, Salekdeh GH: Effects of selenite and tellurite on growth, physiology and proteome of a moderately halophilic bacterium. J Proteome Res 2009, 8:3098-3108.

18. Bajaj M, Schmidt S, Winter J: Formation of Se (0) Nanoparticles by Duganella sp and Agrobacterium sp isolated from Se-laden soil of North-East Punjab, India. Microb Cell Fact 2012, 11:A64.

19. Alain K, Querellou J: Cultivating the uncultured: limits, advances and future challenges. Extremophiles 2009, 13:583-594.

20. Joint I, Mühling M, Querellou J: Culturing marine bacteria- an essential prerequisite for biodiscovery. Microb Biotechnol 2010, 3:564-575.

21. Yuan QL, Yin HY, Nie QL: Nanostructured tellurium semiconductor: from nanoparticles to nanorods. J Exp Nanosci 2013, 8:931-936.

22. Stavila V, Whitmire K, Rusakova I: Synthesis of $\mathrm{Bi}_{2} \mathrm{~S}_{3}$ nanostructures from Bismuth (III) Thiourea and Thiosemicarbazide complexes. Chem Mater 2009, 21:5456-5465.

23. Pages D, Rose J, Conrod S, Cuine S, Carrier P, Heulin T, Achouak W: Heavy metal tolerance in Stenotrophomonas maltophilia. PLoS One 2008, 2:e1539.

24. Kim DH, Kim MG, Jiang S, Lee JH, Hur HG: Promoted reduction of tellurite and formation of extracellular tellurium nanorods by concerted reaction between iron and Shewanella oneidensis MR-1. Environ Sci Technol 2013, 47:8709-8715.

25. Zare B, Faramarzi MA, Sepehrizadeh Z, Shakibaie M, Sassan R, Ahmad RS Biosynthesis and recovery of rod-shaped tellurium nanoparticles and their bactericidal activities. Mater Res Bull 2012, 47:3719-3725.

26. Borghese R, Baccolini C, Francia F, Sabatino P, Turner RJ, Zannoni D: Reduction of chalcogen oxyanions and generation of nanoprecipitatesby the photosynthetic bacterium Rhodobacter capsulatus. $J$ Hazard Mater 2014, 269:24-30.

27. Chasteen TG, Fuentes DE, Tantalean JC, Vasquez CC: Tellurite: history, oxidative stress, and molecular mechanisms of resistance. FEMS Microbiol Rev 2009, 33:820-832.

28. Khan ZH, Khan SA, Salah N, Habib SS, Al-Ghamdi AA: Electrical transport properties of thin film of a-Se ${ }_{87} \mathrm{Te}_{13}$ nanorods. J Exp Nanosci 2011, 6:337-348.

29. Zawadzka AM, Crawford RL, Paszczynski AJ: Pyridine-2,6-Bis (Thiocarboxylic Acid) produced by Pseudomonas stutzeri KC reduces and precipitates selenium and tellurium oxyanions. Appl Environ Microbiol 2006, 72:3119-3129.

30. Kessi J, Hanselmann KW: Similarities between the abiotic reduction of selenite with glutathione and the dissimilatory reaction mediated by Rhodospirillum rubrum and Escherichia coli. J Biol Chem 2004, 279:50662-50669.

31. Madhaiyan M, Poonguzhali S, Saravanan VS, Hari K, Lee KC, Lee JS: Duganella sacchari sp. nov. and Duganella radicis sp. nov., two novel species isolated from rhizosphere of field-grown sugar cane. Int I Syst Evol Microbiol 2013, 63:1126-1131.

32. Ghosh A, Mohod AM, Paknikar KM, Jain RK: Isolation and characterization of selenite- and selenate-tolerant microorganisms from selenium-contaminated sites. World J Microb Biot 2008, 24:1607-1611.

33. Kämpfer $P$, Wellner $S$, Lohse K, Martin K, Lodders N: Duganella phyllosphaerae sp. nov., isolated from the leaf surface of Trifolium repens and proposal to reclassify Duganella violaceinigra into a noval genus as Pseudoduganella violceinigra gen. nov., comb. nov. Syst Appl Microbiol 2012, 35:19-23.

34. Turner RJ, Weiner JH, Taylor DE: Use of diethyldithiocarbamate for quantitative determination of tellurite uptake by bacteria. Anal Biochem 1992, 204:292-295.

35. Miller GL: Use of dinitrosalicylic acid reagent for determination of reducing sugar. Anal Chem 1959, 31:426-428.

\section{Submit your next manuscript to BioMed Central and take full advantage of:}

- Convenient online submission

- Thorough peer review

- No space constraints or color figure charges

- Immediate publication on acceptance

- Inclusion in PubMed, CAS, Scopus and Google Scholar

- Research which is freely available for redistribution

Submit your manuscript at www.biomedcentral.com/submit
C Biomed Central 